\title{
Wave propagation in coated cylinders with reference to fretting fatigue
}

\author{
M RAMESH*, SATISH V KAILAS and K R Y SIMHA \\ Department of Mechanical Engineering, Indian Institute of Science, \\ Bangalore 560012 \\ e-mail:mramesh@mecheng.iisc.ernet.in
}

\begin{abstract}
Fretting fatigue is the phenomenon of crack initiation due to dynamic contact loading, a situation which is commonly encountered in mechanical couplings subjected to vibration. The study of fretting fatigue in high frequency regime has gained importance in recent years. However the stress wave effects at high frequency loading is scanty in the literature. The objective of present investigation is to study stress wave propagation in cylinders with reference to high frequency fretting. The case of a coated cylinder is considered since coating is often provided to improve tribological properties of the component. Rule of mixtures is proposed to understand the dispersion phenomenon in coated or layered cylinder knowing the dispersion relation for the cases of homogeneous cylinders made of coating and substrate materials separately. The possibility of stress wave propagation at the interface with a particular phase velocity without dispersion is also discussed. Results are given for two different thicknesses of coating.
\end{abstract}

Keywords. High frequency fretting; stress waves; frequency equation; layered cylinder.

\section{Introduction}

Fretting fatigue is the phenomenon of crack initiation due to dynamic contact loading. Such situations are commonly encountered whenever materials under contact are subjected to vibration. The frequency of vibration or contact loading is an important parameter. The general frequency regime for fretting fatigue is considered to be $20 \mathrm{~Hz}$ to $20 \mathrm{KHz}$. The literature on fretting fatigue in the high frequency regime is generally limited, except in the recent years. The motivation for studying of fretting fatigue at higher frequency is to investigate the implications of contact dynamics on fretting and fatigue lives and the evolution of coefficient of friction (Matlik \& Farris 2003; Farris et al 2003). Also fretting fatigue tests at high frequencies provide an alternative method for conventional testing by accelerating the testing process, which otherwise would take considerably longer time. Soderberg et al (1986), studied fretting wear behaviour of austenitic stainless steel under ultrasonic frequency for the purpose of

*For correspondence 
accelerating the test and compared the results with that obtained for low frequencies. Rehbein et al (1999), developed high frequency tribometers to study tribological behaviour of friction materials under ultrasonic fretting conditions.

Understanding high frequency fretting fatigue in shafts, rods and piping requires sophisticated elastodynamic techniques of stress analysis. In general, both longitudinal and shear waves are excited in high frequency fretting. The relative importance of these two waves depends on fretting frequency besides geometrical factors and material properties. Thus, predicting initiation and propagation of micro cracks during fretting demands a proper study of stress wave propagation in cylinders and tubes. Pochhammer made fundamental contribution in this area in 1876 (Graff 1975). This analysis is an extension of simple one-dimensional wave propagation in thin bars. Depending on the size of the cylinder and presence of anti fretting coatings if any, stress waves of different frequencies propagate at different phase velocities. The velocity of stress wave propagation determines the rate of energy transport imparted by the dynamic contact loading. Since crack initiation is one of the means of energy dissipation, stress wave characteristics control crack initiation sites and their extension.

\subsection{Wave propagation in cylinders}

Most of the industrial components subjected to fretting have axisymmetric geometry for example shrink-fitted shaft. Hence focus in this work is given to thin rods and cylinders. The equilibrium equation for thin rods whose displacement $u$ at a given axial distance $x$ at time instant $t$ is given by

$$
E \frac{\partial^{2} u}{\partial x^{2}}=\rho \frac{\partial^{2} u}{\partial t^{2}} \Rightarrow \frac{\partial^{2} u}{\partial x^{2}}=\frac{1}{c_{0}^{2}} \frac{\partial^{2} u}{\partial t^{2}},
$$

where $c_{0}=\sqrt{\frac{E}{\rho}}, E$ is the Young's modulus and $\rho$ is the density of the material.

The value $c_{0}$ is a material constant and is called the bar wave speed. Waves of all frequencies travel at this speed in a thin cylinder or rod. However when the cylinder radius is larger, waves of different frequencies travel with different velocities, the characteristics of which will be given by Pochhammer frequency equation. This phenomenon is called as dispersion. Dispersion is not considered in the preliminary analysis for bar wave speed. The bar wave concept applies only to thin cylinders. For finite cylinders the Poisson effect demands Pochhammer analysis. Pochhammer analysis highlights the dispersive behaviour of stress wave propagation in cylinders. Accordingly different frequencies of stress waves propagate at different phase speeds. A plot showing the phase velocity $\left(c_{0}\right)$ as the function of wavenumber $(\xi)$ will provide the dispersive characteristics of wave propagation. The phase velocity $c=\frac{\omega}{\xi}$ where $\omega$ is the angular frequency and $\xi=\frac{2 \pi}{\text { wave length }}$ is the wave number. The phase velocity $c$ corresponds to the velocity at which the wave of wavenumber $\xi$ travels in the solid. Figure 1 shows the dispersive characteristics of a cylinder in terms of normalised phase velocity $\frac{c}{c_{0}}$ as a function of normalised wavenumber $\frac{\xi R}{2 \pi}$ the result given by Pochammer in 1876. In the analysis wave is assumed to propagate only along the axial direction and not in the radial and angular directions. The plot shows that in a cylinder waves of small wavenumber/large wavelength travel at phase velocity $c_{0}$ and waves of large wavenumber/small wavelength travel at a speed $c_{R}$ called the Rayleigh speed. Further, waves of small wavenumber/large wavelengths correspond to waves of low frequency and waves of large wavenumber/small wavelengths 


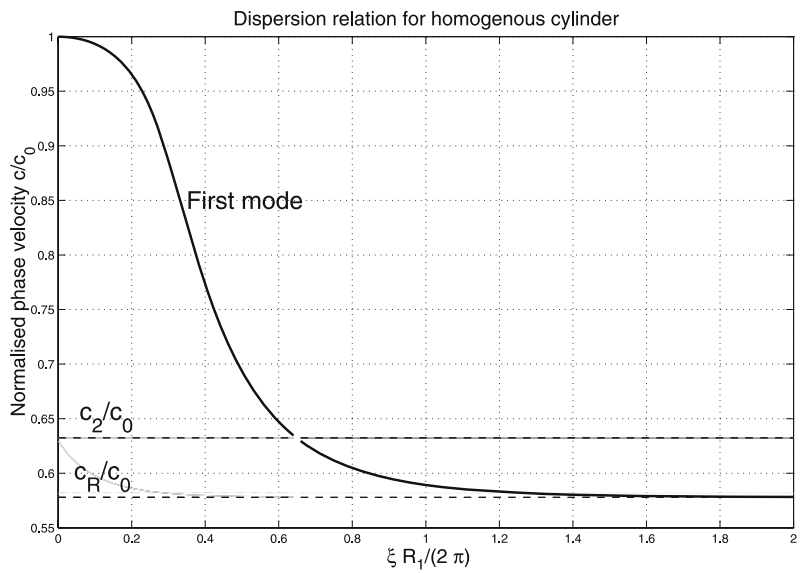

Figure 1. Dispersion relation for homogeneous cylinder.

correspond to waves of high frequency. Hence an equivalent statement can be made as waves of low frequencies propagate at velocity $c_{0}$ while waves of high frequency propagate at velocity $c_{R}$.

Rayleigh wave corresponds to the surface wave propagation in a semi infinite half space subjected to traction free condition at the surface. The speed of Rayleigh wave is the lowest compared to dilatational, shear and bar wave speeds and hence carry more energy. The Rayleigh wave speed $c_{R}$ in a material can be obtained from the relation (Graff 1975)

$$
\left(\frac{c_{R}}{c_{2}}\right)^{6}-8\left(\frac{c_{R}}{c_{2}}\right)^{4}+\left(24-16 k^{-2}\right)\left(\frac{c_{R}}{c_{2}}\right)^{2}-16\left(1-k^{-2}\right)=0,
$$

where $c_{2}=\sqrt{\frac{\mu}{\rho}}$ is the shear wave speed and $k^{2}=\frac{2(1-v)}{1-2 v}$ with $\mu, \rho$ and $v$ being the shear modulus, density and poisson's ratio of the material, respectively. If $v=\frac{1}{4}$ then $\frac{c_{R}}{c_{2}}=$ $\left[2-\frac{2}{\sqrt{3}}\right]^{\frac{1}{2}}=0 \cdot 9194$.

\subsection{Frequency equation for composite cylinders}

Whenever a material has good mechanical properties but poor fretting resistance it is advantageous to provide a layer of material having good fretting resistance instead of changing the entire component material. This extra layer of material can be provided by coating, spraying or by any other surface treatment methods. Okane et al (2003) showed that providing WCCo layer over NiCrMo steel retards fretting crack initiation and reduces crack propagation rate. Similar results were obtained by Nishida et al (2003) for the case of aluminium alloy provided with anodic film. The inhomogeneity introduced by such coating can have significant influence on the wave propagation if the coating is considerably thicker and the material properties differ significantly. Also, presence of any interface in general alters the nature of stress wave propagation.

Whittier \& Jones (1967) studied axisymmetric wave propagation in a two-layered cylinder and compared the results obtained with the shell theory. They also mentioned about possibility of Stonely wave at the interface. They concluded that for the case of large wave numbers the phase velocity is decreasing, and approaches from above the Rayleigh wave velocity of 


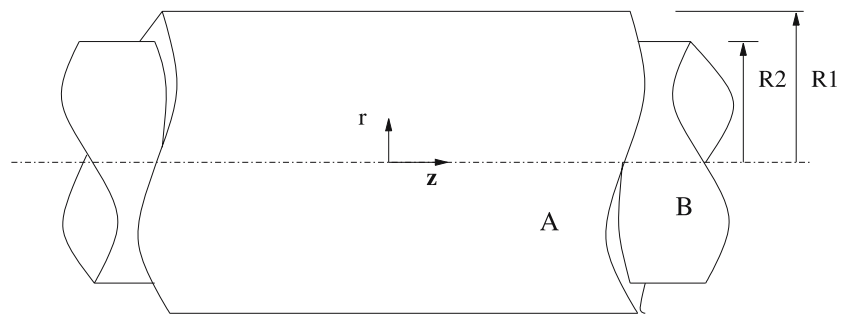

Figure 2. Composite cylinder with coordinate system.

the slower medium. Wave propagation in a coated cylinder is addressed in this investigation (Ramesh et al 2006).

For analysis a cylinder with coating is considered as a composite cylinder with the outer shell made of different material. Let $R_{1}$ and $R_{2}$ are the outer and inner radii of the composite cylinder respectively. The composite cylinder with the corresponding cylindrical coordinate system is shown in figure 2 .

The formulation is similar to that used by Whittier \& Jones (1967) except that inner cylinder is considered to be solid instead of hollow. The mathematical formulation for dynamic stress analysis is obtained using dynamic potentials $\Phi$ and $\Psi$ which are governed by the following equations

$$
\nabla^{2} \Phi=\frac{1}{c_{1}^{2}} \frac{\partial^{2} \Phi}{\partial t^{2}} ; \quad \nabla^{2} \Psi=\frac{1}{c_{2}^{2}} \frac{\partial^{2} \Psi}{\partial t^{2}},
$$

where

$$
\nabla^{2}=\frac{\partial^{2}}{\partial r^{2}}+\frac{1}{r} \frac{\partial}{\partial r}+\frac{\partial^{2}}{\partial z^{2}}
$$

Let $\Delta$ is the dilatation and is given by

$$
\Delta=\frac{\partial u_{r}}{\partial r}+\frac{u_{r}}{r}+\frac{\partial u_{z}}{\partial z}
$$

where $u_{r}$ and $u_{z}$ are the radial and axial displacements.

The following expressions give the stresses and displacements in terms of potential functions.

$$
\begin{aligned}
2 \mu u_{r} & =\frac{\partial \Phi}{\partial r}+\frac{\partial^{2} \Psi}{\partial r \partial z} \\
2 \mu u_{z} & =\frac{\partial \Phi}{\partial z}+\frac{\partial^{2} \Psi}{\partial z^{2}}-\frac{1}{c_{2}^{2}} \frac{\partial^{2} \Psi}{\partial t^{2}} \\
2 \mu \Delta & =\nabla^{2} \Phi \\
\sigma_{r} & =\lambda \Delta+2 \mu \frac{\partial u_{r}}{\partial r} \\
\sigma_{z} & =\lambda \Delta+2 \mu \frac{\partial u_{z}}{\partial z}
\end{aligned}
$$




$$
\begin{aligned}
\sigma_{\theta} & =\lambda \Delta+2 \mu \frac{u_{r}}{r} \\
\tau_{r z} & =\mu\left[\frac{\partial u_{r}}{\partial z}+\frac{\partial u_{z}}{\partial r}\right]
\end{aligned}
$$

Assuming

$$
\begin{aligned}
& \Phi(r, z, t)=\Phi(r, z) \sin (\omega t) \\
& \Psi(r, z, t)=\Psi(r, z) \sin (\omega t) .
\end{aligned}
$$

the time factor $\sin (w t)$ is suppressed in the sequel.

The governing equations 3 become

$$
\nabla^{2} \Phi=-\frac{\omega^{2}}{c_{1}^{2}} \Phi ; \quad \nabla^{2} \Psi=-\frac{\omega^{2}}{c_{2}^{2}} \Psi .
$$

The method of Fourier integral transform is used to solve for $\Phi$ and $\Psi$. Considering the case of symmetry of $\sigma_{r}$ and anti symmetry of $\tau_{r z}$ about $z=0$ the dynamic potentials $\Phi$ and $\Psi$ are chosen to be even and odd functions respectively. Hence Fourier cosine transform is applied over $\Phi$ and Fourier sine transform is applied over $\Psi$ as given below.

$$
\begin{aligned}
& \bar{\Phi}(r, \xi)=\int_{0}^{\infty} \Phi(r, z) \cos (\xi z) d z \\
& \bar{\Psi}(r, \xi)=\int_{0}^{\infty} \Psi(r, z) \sin (\xi z) d z .
\end{aligned}
$$

The inverse transforms are:

$$
\begin{aligned}
& \Phi(r, z)=\frac{2}{\pi} \int_{0}^{\infty} \bar{\Phi}(r, \xi) \cos (\xi z) d \xi \\
& \Psi(r, z)=\frac{2}{\pi} \int_{0}^{\infty} \bar{\Psi}(r, \xi) \sin (\xi z) d \xi .
\end{aligned}
$$

The stresses and displacements in the Fourier space are:

$$
\begin{aligned}
2 \mu \bar{u}_{r} & =\frac{d \bar{\Phi}}{d r}+\xi \frac{d \bar{\Psi}}{d r} \\
2 \mu \bar{u}_{z} & =-\xi \bar{\Phi}+\left(\frac{\omega^{2}}{c_{2}^{2}}-\xi^{2}\right) \bar{\Psi} \\
2 \mu \bar{\Delta} & =-\frac{\omega^{2}}{c_{1}^{2}} \bar{\Phi} \\
\bar{\sigma}_{r} & =-\frac{\lambda}{2 \mu} \frac{\omega^{2}}{c_{1}^{2}} \bar{\Phi}+\frac{d^{2} \bar{\Phi}}{d r^{2}}+\xi \frac{d^{2} \bar{\Psi}}{d r^{2}}
\end{aligned}
$$




$$
\begin{aligned}
& \bar{\tau}_{r z}=-\xi \frac{d \Phi}{d r}+\frac{1}{2}\left(\frac{\omega^{2}}{c_{2}^{2}}-2 \xi^{2}\right) \frac{d \bar{\Psi}}{d r} \\
& \bar{\sigma}_{\theta}=-\frac{\lambda}{2 \mu} \frac{\omega^{2}}{c_{1}^{2}} \bar{\Phi}+\frac{1}{r}\left[\frac{d \bar{\Phi}}{d r}+\xi \frac{d \bar{\Psi}}{d r}\right] \\
& \bar{\sigma}_{z}=-\frac{\lambda}{2 \mu} \frac{\omega^{2}}{c_{1}^{2}} \bar{\Phi}+\xi\left[-\xi \bar{\Phi}+\left(\frac{\omega^{2}}{c_{2}^{2}}-\xi^{2}\right) \bar{\Psi}\right] .
\end{aligned}
$$

Applying the respective transform over the governing equations of the potentials yields the following ordinary differential equations.

$$
\begin{aligned}
& \frac{d^{2} \bar{\Phi}}{d r^{2}}+\frac{1}{r} \frac{d \bar{\Phi}}{d r}+\left(\frac{\omega^{2}}{c_{1}^{2}}-\xi^{2}\right) \bar{\Phi}=0 \\
& \frac{d^{2} \bar{\Psi}}{d r^{2}}+\frac{1}{r} \frac{d \bar{\Psi}}{d r}+\left(\frac{\omega^{2}}{c_{2}^{2}}-\xi^{2}\right) \bar{\Psi}=0
\end{aligned}
$$

The solution to the above equations corresponding to outer layer of cylinder made of material $A$ is

Case (i)

$$
\begin{aligned}
& \text { if } 0<\xi^{2}<\frac{\omega^{2}}{c_{1 A}^{2}} \text { then } \alpha_{A}^{2}=\frac{\omega^{2}}{c_{1 A}^{2}}-\xi^{2}, \beta_{A}^{2}=\frac{\omega^{2}}{c_{2 A}^{2}}-\xi^{2} \\
& \bar{\Phi}_{A}=A_{1}(\xi) J_{0}\left(\alpha_{A} r\right)+A_{2}(\xi) Y_{0}\left(\alpha_{A} r\right) \\
& \bar{\Psi}_{A}=\frac{A_{3}(\xi)}{\beta_{A}} J_{0}\left(\beta_{A} r\right)+\frac{A_{4}(\xi)}{\beta_{A}} Y_{0}\left(\beta_{A} r\right) .
\end{aligned}
$$

Case (ii)

$$
\text { if } \begin{array}{r}
\frac{\omega^{2}}{c_{1 A}^{2}}<\xi^{2}<\frac{\omega^{2}}{c_{2 A}^{2}} \text { then } \alpha_{A}^{2}=\xi^{2}-\frac{\omega^{2}}{c_{1 A}^{2}}, \beta_{A}^{2}=\frac{\omega^{2}}{c_{2 A}^{2}}-\xi^{2} \\
\bar{\Phi}_{A}=A_{1}(\xi) I_{0}\left(\alpha_{A} r\right)+A_{2}(\xi) K_{0}\left(\alpha_{A} r\right) \\
\bar{\Psi}_{A}=\frac{A_{3}(\xi)}{\beta_{A}} J_{0}\left(\beta_{A} r\right)+\frac{A_{4}(\xi)}{\beta_{A}} Y_{0}\left(\beta_{A} r\right) .
\end{array}
$$

Case (iii)

if $\frac{\omega^{2}}{c_{1 A}^{2}}<\xi^{2}<\infty$ then $\alpha_{A}^{2}=\xi^{2}-\frac{\omega^{2}}{c_{1 A}^{2}}, \beta_{A}^{2}=\xi^{2}-\frac{\omega^{2}}{c_{2 A}^{2}}$

$$
\bar{\Phi}_{A}=A_{1}(\xi) I_{0}\left(\alpha_{A} r\right)+A_{2}(\xi) K_{0}\left(\alpha_{A} r\right)
$$




$$
\bar{\Psi}_{A}=\frac{A_{3}(\xi)}{\beta_{A}} I_{0}\left(\beta_{A} r\right)+\frac{A_{4}(\xi)}{\beta_{A}} K_{0}\left(\beta_{A} r\right)
$$

where $c_{1 A}=\sqrt{\frac{\lambda_{A}+2 \mu_{A}}{\rho_{A}}}$ and $c_{2 A}=\sqrt{\frac{\mu}{\rho}}$ are the dilatational and shear wave speeds respectively in material $A$. The functions $J_{0}, Y_{0}, I_{0}$ and $K_{0}$ are the Bessel functions. The solution for inner core made of material $B$ is obtained by replacing suffix $A$ by $B$. The constants $B_{2}=B_{4}=0$ the core being solid. For the case of homogeneous cylinder made of material $A$ which corresponds to the Pochhammer problem $A_{2}=A_{4}=0$. However, a general case of coated cylinder requires six non-zero constants.

Using this solution for $\Phi_{A}, \Phi_{B}, \Psi_{A}$ and $\Psi_{B}$ the stresses and displacements in the Fourier space are:

Cylinder $A$ (coating):

$$
\begin{aligned}
2 \mu_{A} \bar{u}_{r A} & =A_{1}(\xi) a_{11}(r)+A_{2}(\xi) a_{12}(r)+A_{3}(\xi) a_{13}(r)+A_{4}(\xi) a_{14}(r) \\
2 \mu_{A} \bar{u}_{z A} & =A_{1}(\xi) a_{21}(r)+A_{2}(\xi) a_{22}(r)+A_{3}(\xi) a_{23}(r)+A_{4}(\xi) a_{24}(r) \\
\bar{\sigma}_{r A} & =A_{1}(\xi) a_{31}(r)+A_{2}(\xi) a_{32}(r)+A_{3}(\xi) a_{33}(r)+A_{4}(\xi) a_{34}(r) \\
\bar{\tau}_{r z A} & =A_{1}(\xi) a_{41}(r)+A_{2}(\xi) a_{42}(r)+A_{3}(\xi) a_{43}(r)+A_{4}(\xi) a_{44}(r) \\
\bar{\sigma}_{\theta_{A}} & =A_{1}(\xi) a_{51}(r)+A_{2}(\xi) a_{52}(r)+A_{3}(\xi) a_{53}(r)+A_{4}(\xi) a_{54}(r) \\
\bar{\sigma}_{z A} & =A_{1}(\xi) a_{61}(r)+A_{2}(\xi) a_{62}(r)+A_{3}(\xi) a_{63}(r)+A_{4}(\xi) a_{64}(r) .
\end{aligned}
$$

Cylinder $B$ (core):

$$
\begin{aligned}
2 \mu_{B} \bar{u}_{r B} & =B_{1}(\xi) b_{11}(r)+B_{3}(\xi) b_{13}(r) \\
2 \mu_{B} \bar{u}_{z B} & =B_{1}(\xi) b_{21}(r)+B_{3}(\xi) b_{23}(r) \\
\bar{\sigma}_{r B} & =B_{1}(\xi) b_{31}(r)+B_{3}(\xi) b_{33}(r) \\
\bar{\tau}_{r z B} & =B_{1}(\xi) b_{41}(r)+B_{3}(\xi) b_{43}(r) \\
\bar{\sigma}_{\theta B} & =B_{1}(\xi) b_{51}(r)+B_{3}(\xi) b_{53}(r) \\
\bar{\sigma}_{z B} & =B_{1}(\xi) b_{61}(r)+B_{3}(\xi) b_{63}(r) .
\end{aligned}
$$

The expressions for $a_{i j}$ and $b_{i j}$ are given in Appendix A. The boundary conditions for the frequency equation of homogeneous cylinder is that $\sigma_{r}=\tau_{r z}=0$ at $r=R$, where $\mathrm{R}$ is the radius of cylinder. For the case of composite cylinder the stress and displacement continuity has to be ensured at the interface along with the traction free surface condition.

Substituting the expressions for the transformed stresses and displacements from equations 35 and 36 in the above mentioned boundary conditions we get the following equation in the matrix form

$$
[\mathrm{M}]_{6 \times 6}[\mathrm{~A}]_{6 \times 1}=[0]_{6 \times 1}
$$


where

$$
\begin{aligned}
& \mathbf{M}=\left[\begin{array}{cccccc}
a_{31}\left(R_{1}\right) & a_{32}\left(R_{1}\right) & a_{33}\left(R_{1}\right) & a_{34}\left(R_{1}\right) & 0 & 0 \\
a_{41}\left(R_{1}\right) & a_{42}\left(R_{1}\right) & a_{43}\left(R_{1}\right) & a_{44}\left(R_{1}\right) & 0 & 0 \\
a_{31}\left(R_{2}\right) & a_{32}\left(R_{2}\right) & a_{33}\left(R_{2}\right) & a_{34}\left(R_{2}\right) & -b_{31}\left(R_{2}\right) & -b_{33}\left(R_{2}\right) \\
a_{41}\left(R_{2}\right) & a_{42}\left(R_{2}\right) & a_{43}\left(R_{2}\right) & a_{44}\left(R_{2}\right) & -b_{41}\left(R_{2}\right) & -b_{43}\left(R_{2}\right) \\
a_{11}\left(R_{2}\right) & a_{12}\left(R_{2}\right) & a_{13}\left(R_{2}\right) & a_{14}\left(R_{2}\right) & -\frac{\mu_{A}}{\mu_{B}} b_{11}\left(R_{2}\right) & -\frac{\mu_{A}}{\mu_{B}} b_{13}\left(R_{2}\right) \\
a_{21}\left(R_{2}\right) & a_{22}\left(R_{2}\right) & a_{23}\left(R_{2}\right) & a_{24}\left(R_{2}\right) & -\frac{\mu_{A}}{\mu_{B}} b_{21}\left(R_{2}\right) & -\frac{\mu_{A}}{\mu_{B}} b_{23}\left(R_{2}\right)
\end{array}\right] \\
& \mathbf{A}=\left[\begin{array}{c}
A_{1} \\
A_{2} \\
A_{3} \\
A_{4} \\
B_{1} \\
B_{3}
\end{array}\right] ; \quad \mathbf{0}=\left[\begin{array}{l}
0 \\
0 \\
0 \\
0 \\
0 \\
0
\end{array}\right]
\end{aligned}
$$

where $\mathbf{A}$ is the column vector with the constants $A_{j}$ and $B_{j}$ as the elements, matrix $\mathbf{M}$ consists of the coefficients of these constants as the elements and $\mathbf{0}$ represents the zero column vector. The frequency equation is now given by $\operatorname{Det}(\mathbf{M})=0$. For the homogeneous case the frequency equation (Pochhammer frequency equation (Graff 1975)) becomes

$$
\begin{aligned}
& \frac{2 \alpha}{R}\left(\beta^{2}+\xi^{2}\right) J_{1}(\alpha R) J_{1}(\beta R)-\left(\beta^{2}-\xi^{2}\right)^{2} J_{0}(\alpha R) J_{1}\left(\beta_{R}\right) \\
& \quad-4 \xi^{2} \alpha \beta J_{1}(\alpha R) J_{0}(\beta R)=0 .
\end{aligned}
$$

The expression for composite cylinder case being more complicated is not shown here. However frequency equation for the case of composite cylinder is solved numerically and the results are compared with that of the homogeneous case.

\section{Results and discussion}

The dispersion characteristics for the case of homogeneous cylinder (figure 1) shows that at lower wave number and hence at lower frequencies the propagation speed corresponds to that of bar wave speed. However at higher wave number and hence at higher frequencies the speed of wave propagation is same as that of Rayleigh wave speed. This shows that at higher frequencies the wave propagation and the energy transfer occur predominately at the surface. Since fretting being a near surface phenomenon these surface waves will have considerable effect on the fretting fatigue. For the case of composite cylinder or coated cylinder different combinations of material properties are chosen. If $\lambda_{A}=\mu_{A}$ and $\lambda_{B}=\mu_{B}$ then Poisson's ratio $v_{A}=v_{B}=1 / 4$. Also density of both the materials is taken to be equal to $2500 \mathrm{Kg} / \mathrm{m}^{3}$. The value of $\mu_{B}$ is taken to be $25 \times 10^{9}$. Results are shown for the cases of $\lambda_{A}=\lambda_{B}, 2 \lambda_{B}$ 


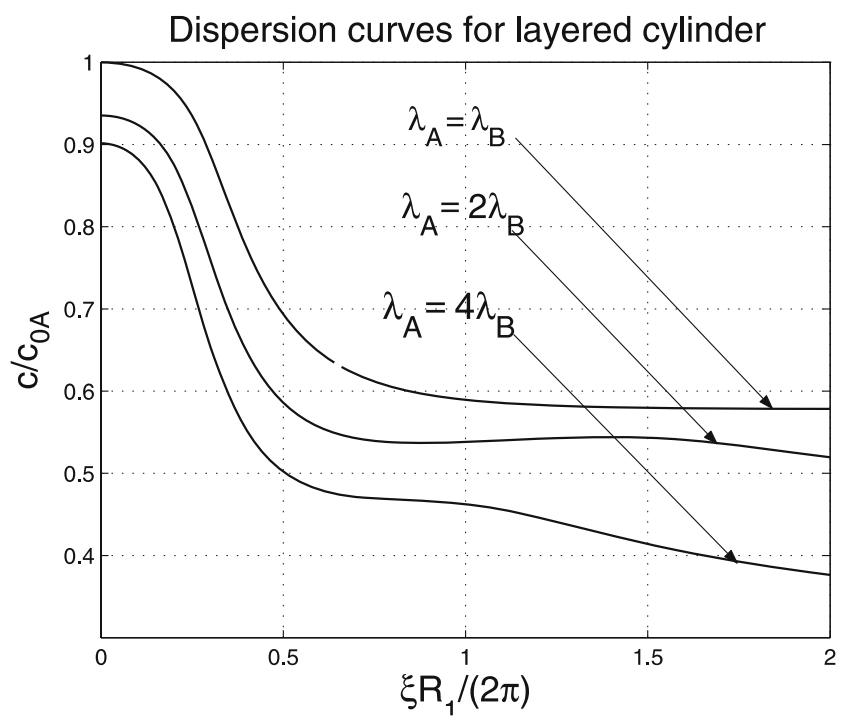

Figure 3. Effect of elastic mismatch between outer layer and inner core material.

and $4 \lambda_{B}$, the interface radius $R_{2}=0.5$ (figure 3 ). The phase velocity is normalised with the bar wave speed of the coating. The normalised phase velocity $c / c_{0 A}$ is plotted with respect to the normalised wave number $\bar{\xi}=\frac{\xi R_{1}}{2 \pi}$. The nature of plot for the case of coated cylinder is similar to that of homogeneous cylinder except for two reasons. First one is that the plot is shifted down by almost a constant value depending upon the ratio $\lambda_{A} / \lambda_{B}$. Also they have small maxima and minima whereas the plot for homogeneous case monotonically decreases to Rayleigh wave speed of the material.

This similarity in the nature of the curves infers that the approximate plot for the case of composite cylinder can be obtained from the plots corresponding to substrate and coating material by using the rule of mixture (figure 4). The rule of mixture can be appropriately stated as

$$
c_{\text {composite }}=x c_{\text {substrate }}+(1-x) c_{\text {coating }} \text {, }
$$

where $x=\frac{R_{2}^{2}}{R_{1}^{2}}$

$c_{\text {composite }}=$ Phase velocity in the coated cylinder of outer radius $R_{1}$ and interface radius $R_{2}$ $c_{\text {coating }}=$ Phase velocity in a homogeneous cylinder of radius $R_{1}$ completely made of coating material $A$

$c_{\text {substrate }}=$ Phase velocity in a homogeneous cylinder of radius $R_{1}$ completely made of substrate (core cylinder) material $B$.

Figure 4 shows the plot of $\frac{c}{c_{0 A}}$ vs $\bar{\xi}$ for the cases of composite, substrate and coating. Also the plot for the composite calculated from the rule of mixtures is shown in dotted lines.

The plot shows that the prediction by rule of mixtures is good for the values of $\xi R_{1} /(2 \pi)$ near zero and near 1.5 . In the intermediate region prediction by rule of mixtures is still fair. However the rule of mixture fails to predict the small oscillation near $\frac{\xi R_{1}}{2 \pi}>1$ in the dispersion curve of composite cylinder. Figure 5 shows the slope of dispersion curves plotted in figure 4. The plot shows that the dispersion curve of composite cylinder crosses zero at two points near $\frac{\xi R_{1}}{2 \pi}=0.9$ and $\frac{\xi R_{1}}{2 \pi}=1.4$ which is not seen in the other two cases. This is important because 


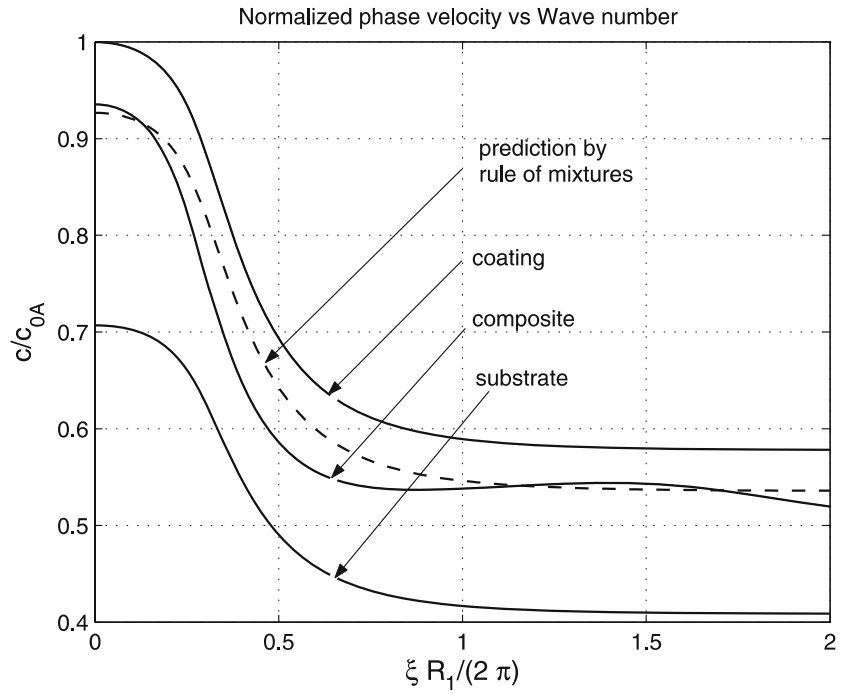

Figure 4. Prediction by rule of mixtures.

the group velocity depends on the slope of dispersion curve. Also this implies that there is a possible case of wave travelling with a particular phase velocity without dispersion.

The group velocity $\left(c_{g}\right)$ corresponds to the velocity at which the energy of the wave is being transmitted. The expression for $c_{g}$ shows that $c_{g}=\frac{d \omega}{d \xi}=c+\xi \frac{d c}{d \xi}$ because $\omega=c \xi$. Thus $c_{g}$ and hence the rate of energy transmission depends on the slope of dispersion curve $\frac{d c}{d \xi}$. In figure 6 group velocity of composite is plotted along with that of homogeneous cases made of the coating and core materials. The plot shows that behaviour of $c_{g}$ is very different for composite cylinder compared to the homogeneous cases. Hence the rule of mixture will not be able to predict the $c_{g}$ of composite cylinders even though it predicts the dispersion curve fairly well. Further, it should be noted that the $c_{g}$ curve for composite cylinder oscillates whose amplitude is contained between the two curves correspond to the homogeneous cases made of materials $A$ and $B$.

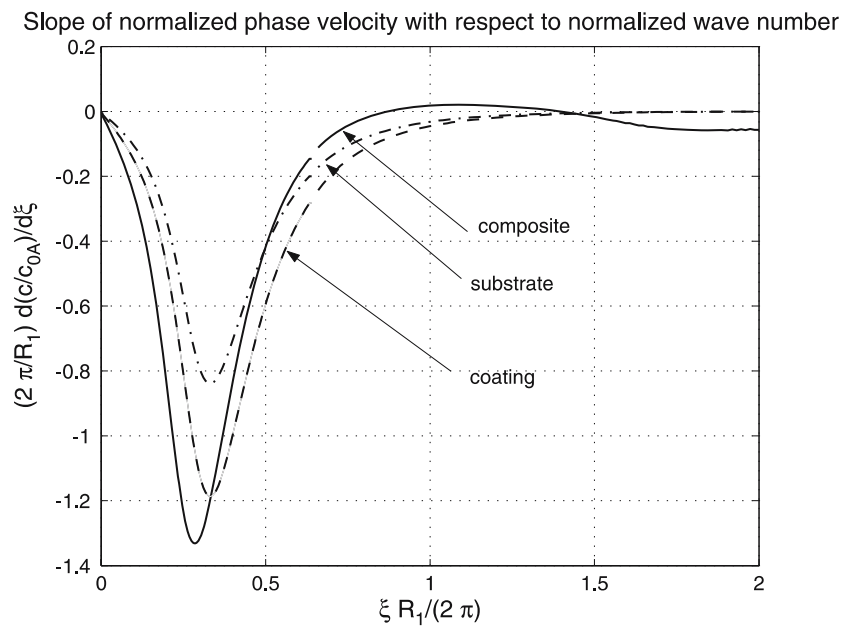

Figure 5. Gradient of phase speed curve. 


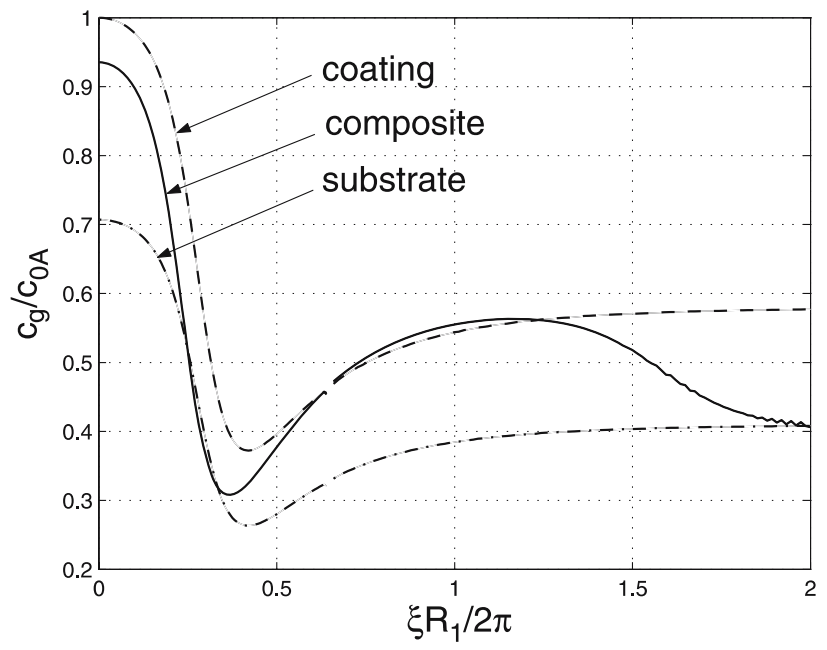

Figure 6. Group speed Vs Wave number.

The dispersion curve for coated cylinder is shown in figure 7 for the case of interface radius being $0.8 R_{1}$ and $0.9 R_{1}$. The plot shows that the dispersion relation becomes similar to that of the core as the thickness of coating is reduced. This can be well explained by the rule of mixture.

\section{Conclusion}

The elastodynamics of high frequency fretting in coated or layered cylinder is investigated. The effect of mismatch between elastic properties of the coating and substrate or core on dispersion is presented. The rule of mixtures is proposed to understand the dispersion relation in layered cylinder, whose predictions are good both at lower and higher values of wave number except at the intermediate range, when compared with the actual dispersion relation for layered cylinder. The possibility of phase velocity without dispersion at the interface is

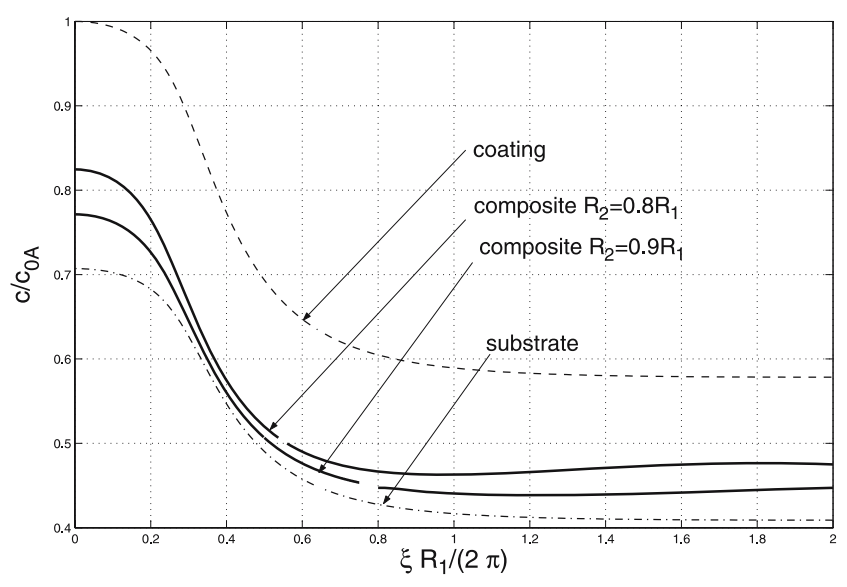

Figure 7. Effect of coating thickness. 
demonstrated however the corresponding range of wave number is small. Results for dispersion relation are shown for the cases of interface radius lying closer to the outer surface. The dispersion relation approaches the dispersion relation corresponding to that of the inner core as the interface radius becomes closer and closer to the surface. Since stress wave propagation and crack initiation are phenomena of energy transfer and dissipation a greater interaction is expected between them. Thus, at higher frequencies the contact dynamics and stress wave propagation will play a major role in fretting. Especially for the case where a thick coating is present the dynamic analysis becomes inevitable.

\section{Appendix A}

Appendix A.1. Expressions for $a_{i j}$

Case (i)

$$
\begin{aligned}
0<\xi<\frac{\omega}{c_{1 A}}< & \frac{\omega}{c_{2 A}}<\infty \\
a_{11} & =-\alpha_{A} J_{1}\left(\alpha_{A} r\right) \\
a_{12} & =-\alpha_{A} Y_{1}\left(\alpha_{A} r\right) \\
a_{13} & =-\xi J_{1}\left(\beta_{A} r\right) \\
a_{14} & =-\xi Y_{1}\left(\beta_{A} r\right) \\
a_{21} & =-\xi J_{0}\left(\alpha_{A} r\right) \\
a_{22} & =-\xi Y_{0}\left(\alpha_{A} r\right) \\
a_{23} & =\beta_{A} J_{0}\left(\beta_{A} r\right) \\
a_{24} & =\beta_{A} Y_{0}\left(\beta_{A} r\right) \\
a_{31} & =-\left[\left(\frac{\lambda_{A}}{2 \mu_{A}}+1\right) \alpha_{A}^{2}+\frac{\lambda_{A}}{2 \mu_{A}} \xi^{2}\right] J_{0}\left(\alpha_{A} r\right)+\alpha_{A}^{2} \frac{J_{1}\left(\alpha_{A} r\right)}{\alpha_{A} r} \\
a_{41} & =\xi \alpha_{A} J_{1}\left(\alpha_{A} r\right) \\
a_{32} & =-\left[\left(\frac{\lambda_{A}}{2 \mu_{A}}+1\right) \alpha_{A}^{2}+\frac{\lambda_{A}}{2 \mu_{A}} \xi^{2}\right] Y_{0}\left(\alpha_{A} r\right)+\alpha_{A}^{2} \frac{Y_{1}\left(\alpha_{A} r\right)}{\alpha_{A} r} \\
a_{33} & =\xi \beta_{A}\left[-J_{0}\left(\beta_{A} r\right)+\frac{J_{1}\left(\beta_{A} r\right)}{\beta_{A} r}\right] \\
a_{A} r & Y_{1}\left(\beta_{A} r\right) \\
&
\end{aligned}
$$




$$
\begin{aligned}
& a_{42}=\xi \alpha_{A} Y_{1}\left(\alpha_{A} r\right) \\
& a_{43}=-\frac{1}{2}\left(\beta_{A}^{2}-\xi^{2}\right) J_{1}\left(\beta_{A} r\right) \\
& a_{44}=-\frac{1}{2}\left(\beta_{A}^{2}-\xi^{2}\right) Y_{1}\left(\beta_{A} r\right) \\
& a_{51}=-\frac{\lambda_{A}}{2 \mu_{A}}\left(\alpha_{A}^{2}+\xi^{2}\right) J_{0}\left(\alpha_{A} r\right)-\alpha_{A}^{2} \frac{J_{1}\left(\alpha_{A} r\right)}{\alpha_{A} r} \\
& a_{52}=-\frac{\lambda_{A}}{2 \mu_{A}}\left(\alpha_{A}^{2}+\xi^{2}\right) Y_{0}\left(\alpha_{A} r\right)-\alpha_{A}^{2} \frac{Y_{1}\left(\alpha_{A} r\right)}{\alpha_{A} r} \\
& a_{53}=-\xi \beta_{A} \frac{J_{1}\left(\beta_{A} r\right)}{\beta_{A} r} \\
& a_{54}=-\xi \beta_{A} \frac{Y_{1}\left(\beta_{A} r\right)}{\beta_{A} r} \\
& a_{61}=-\left[\frac{\lambda_{A}}{2 \mu_{A}} \alpha_{A}^{2}+\left(\frac{\lambda_{A}}{2 \mu_{A}}+1\right) \xi^{2}\right] J_{0}\left(\alpha_{A} r\right) \\
& a_{62}=-\left[\frac{\lambda_{A}}{2 \mu_{A}} \alpha_{A}^{2}+\left(\frac{\lambda_{A}}{2 \mu_{A}}+1\right) \xi^{2}\right] Y_{0}\left(\alpha_{A} r\right) \\
& a_{64}=\xi \beta_{A} J_{0}\left(\beta_{A} r\right) \\
& \beta_{A} Y_{0}\left(\beta_{A} r\right) .
\end{aligned}
$$

Case (ii)

$$
\begin{aligned}
0<\frac{\omega}{c_{1 A}}<\xi<\frac{\omega}{c_{2 A}}<\infty \\
a_{11}=\alpha_{A} I_{1}\left(\alpha_{A} r\right) \\
a_{12}=-\alpha_{A} K_{1}\left(\alpha_{A} r\right) \\
a_{13}=-\xi J_{1}\left(\beta_{A} r\right) \\
a_{14}=-\xi Y_{1}\left(\beta_{A} r\right) \\
a_{21}=-\xi I_{0}\left(\alpha_{A} r\right) \\
a_{22}=-\xi K_{0}\left(\alpha_{A} r\right) \\
a_{23}=\beta_{A} J_{0}\left(\beta_{A} r\right) \\
a_{24}=\beta_{A} Y_{0}\left(\beta_{A} r\right)
\end{aligned}
$$




$$
\begin{aligned}
& a_{31}=\left[\left(\frac{\lambda_{A}}{2 \mu_{A}}+1\right) \alpha_{A}^{2}-\frac{\lambda_{A}}{2 \mu_{A}} \xi^{2}\right] I_{0}\left(\alpha_{A} r\right)-\alpha_{A}^{2} \frac{I_{1}\left(\alpha_{A} r\right)}{\alpha_{A} r} \\
& a_{32}=\left[\left(\frac{\lambda_{A}}{2 \mu_{A}}+1\right) \alpha_{A}^{2}-\frac{\lambda_{A}}{2 \mu_{A}} \xi^{2}\right] K_{0}\left(\alpha_{A} r\right)+\alpha_{A}^{2} \frac{K_{1}\left(\alpha_{A} r\right)}{\alpha_{A} r} \\
& a_{33}=\xi \beta_{A}\left[-J_{0}\left(\beta_{A} r\right)+\frac{J_{1}\left(\beta_{A} r\right)}{\beta_{A} r}\right] \\
& a_{34}=\xi \beta_{A}\left[-Y_{0}\left(\beta_{A} r\right)+\frac{Y_{1}\left(\beta_{A} r\right)}{\beta_{A} r}\right] \\
& a_{41}=-\xi \alpha_{A} I_{1}\left(\alpha_{A} r\right) \\
& a_{42}=\xi \alpha_{A} K_{1}\left(\alpha_{A} r\right) \\
& a_{43}=-\frac{1}{2}\left(\beta_{A}^{2}-\xi^{2}\right) J_{1}\left(\beta_{A} r\right) \\
& a_{44}=-\frac{1}{2}\left(\beta_{A}^{2}-\xi^{2}\right) Y_{1}\left(\beta_{A} r\right) \\
& a_{51}=\frac{\lambda_{A}}{2 \mu_{A}}\left(\alpha_{A}^{2}-\xi^{2}\right) I_{0}\left(\alpha_{A} r\right)+\alpha_{A}^{2} \frac{I_{1}\left(\alpha_{A} r\right)}{\alpha_{A} r} \\
& a_{52}=\frac{\lambda_{A}}{2 \mu_{A}}\left(\alpha_{A}^{2}-\xi^{2}\right) K_{0}\left(\alpha_{A} r\right)-\alpha_{A}^{2} \frac{K_{1}\left(\alpha_{A} r\right)}{\alpha_{A} r} \\
& a_{53}=-\xi \beta_{A} \frac{J_{1}\left(\beta_{A} r\right)}{\beta_{A} r} \\
& a_{54}=-\xi \beta_{A} \frac{Y_{1}\left(\beta_{A} r\right)}{\beta_{A} r} \\
& a_{61}=\left[\frac{\lambda_{A}}{2 \mu_{A}} \alpha_{A}^{2}-\left(\frac{\lambda_{A}}{2 \mu_{A}}+1\right) \xi^{2}\right] I_{0}\left(\alpha_{A} r\right) \\
& a_{62}=\left[\frac{\lambda_{A}}{2 \mu_{A}} \alpha_{A}^{2}-\left(\frac{\lambda_{A}}{2 \mu_{A}}+1\right) \xi^{2}\right] K_{0}\left(\alpha_{A} r\right) \\
& a_{63}=\xi \beta_{A} J_{0}\left(\beta_{A} r\right) \\
& a_{64}=\xi \beta_{A} Y_{0}\left(\beta_{A} r\right) \text {. }
\end{aligned}
$$

Case (iii)

$$
\begin{aligned}
0<\frac{\omega}{c_{1 A}}<\frac{\omega}{c_{2 A}}<\xi<\infty \\
a_{11}=\alpha_{A} I_{1}\left(\alpha_{A} r\right)
\end{aligned}
$$




$$
\begin{aligned}
& a_{12}=-\alpha_{A} K_{1}\left(\alpha_{A} r\right) \\
& a_{13}=\xi I_{1}\left(\beta_{A} r\right) \\
& a_{14}=-\xi K_{1}\left(\beta_{A} r\right) \\
& a_{21}=-\xi I_{0}\left(\alpha_{A} r\right) \\
& a_{22}=-\xi K_{0}\left(\alpha_{A} r\right) \\
& a_{23}=-\beta_{A} I_{0}\left(\beta_{A} r\right) \\
& a_{24}=-\beta_{A} K_{0}\left(\beta_{A} r\right) \\
& a_{31}=\left[\left(\frac{\lambda_{A}}{2 \mu_{A}}+1\right) \alpha_{A}^{2}-\frac{\lambda_{A}}{2 \mu_{A}} \xi^{2}\right] I_{0}\left(\alpha_{A} r\right)-\alpha_{A}^{2} \frac{I_{1}\left(\alpha_{A} r\right)}{\alpha_{A} r} \\
& a_{54} \\
& a_{52}=-\xi \beta_{A} \frac{K_{1}\left(\beta_{A} r\right)}{\beta_{A} r}\left(\frac{\lambda_{A}}{2 \mu_{A}}\left(\alpha_{A}^{2}-\xi_{A}^{2}\right) K_{0}\left(\alpha_{A} r\right)-\alpha_{A}^{2} \frac{K_{1}\left(\alpha_{A} r\right)}{\alpha_{A} r}\right. \\
& a_{51}=\frac{I_{A}\left(\beta_{A} r\right)}{2 \mu_{A}}\left(\alpha_{A}^{2}-\xi^{2}\right) I_{0}\left(\alpha_{A} r\right)+\alpha_{A}^{2} \frac{I_{1}\left(\alpha_{A} r\right)}{\alpha_{A} r} \\
& a_{43}=-\frac{1}{2}\left(\beta_{A}^{2}+\xi_{A}^{2}\right) I_{1}\left(\beta_{A} r\right)+\alpha_{A}^{2} \frac{K_{1}\left(\alpha_{A} r\right)}{\alpha_{A} r} \\
& a_{33}=\xi \beta_{A}\left[I_{0}\left(\beta_{A} r\right)-\frac{I_{1}\left(\beta_{A} r\right)}{\beta_{A} r}\right] \\
& a_{42}=-\xi \alpha_{A} I_{1}\left(\alpha_{A} r\right) \\
& a_{34}=\xi \beta_{A}\left[K_{0}\left(\beta_{A} r\right)+\frac{K_{1}\left(\beta_{A} r\right)}{\beta_{A} r}\right]
\end{aligned}
$$




$$
\begin{aligned}
& a_{61}=\left[\frac{\lambda_{A}}{2 \mu_{A}} \alpha_{A}^{2}-\left(\frac{\lambda_{A}}{2 \mu_{A}}+1\right) \xi^{2}\right] I_{0}\left(\alpha_{A} r\right) \\
& a_{62}=\left[\frac{\lambda_{A}}{2 \mu_{A}} \alpha_{A}^{2}-\left(\frac{\lambda_{A}}{2 \mu_{A}}+1\right) \xi^{2}\right] K_{0}\left(\alpha_{A} r\right) \\
& a_{63}=-\xi \beta_{A} I_{0}\left(\beta_{A} r\right) \\
& a_{64}=-\xi \beta_{A} K_{0}\left(\beta_{A} r\right) .
\end{aligned}
$$

Appendix A.2. Expressions for $b_{i j}$

Case (i)

$$
\begin{aligned}
& 0<\xi<\frac{\omega}{c_{1 B}}<\frac{\omega}{c_{2 B}}<\infty \\
& b_{11}=-\alpha_{B} J_{1}\left(\alpha_{B} r\right) \\
& b_{13}=-\xi J_{1}\left(\beta_{B} r\right) \\
& b_{21}=-\xi J_{0}\left(\alpha_{B} r\right) \\
& b_{23}=\beta_{B} J_{0}\left(\beta_{B} r\right) \\
& b_{31}=-\left[\left(\frac{\lambda_{B}}{2 \mu_{B}}+1\right) \alpha_{B}^{2}+\frac{\lambda_{B}}{2 \mu_{B}} \xi^{2}\right] J_{0}\left(\alpha_{B} r\right)+\alpha_{B}^{2} \frac{J_{1}\left(\alpha_{B} r\right)}{\alpha_{B} r} \\
& b_{33}=\xi \beta_{B}\left[-J_{0}\left(\beta_{B} r\right)+\frac{J_{1}\left(\beta_{B} r\right)}{\beta_{B} r}\right] \\
& b_{41}=\xi \alpha_{B} J_{1}\left(\alpha_{B} r\right) \\
& b_{43}=-\frac{1}{2}\left(\beta_{B}^{2}-\xi^{2}\right) J_{1}\left(\beta_{B} r\right) \\
& b_{51}=-\frac{\lambda_{B}}{2 \mu_{B}}\left(\alpha_{B}^{2}+\xi^{2}\right) J_{0}\left(\alpha_{B} r\right)-\alpha_{B}^{2} \frac{J_{1}\left(\alpha_{B} r\right)}{\alpha_{B} r} \\
& b_{53}=-\xi \beta_{B} \frac{J_{1}\left(\beta_{B} r\right)}{\beta_{B} r} \\
& b_{61}=-\left[\frac{\lambda_{B}}{2 \mu_{B}} \alpha_{B}^{2}+\left(\frac{\lambda_{B}}{2 \mu_{B}}+1\right) \xi^{2}\right] J_{0}\left(\alpha_{B} r\right) \\
& b_{63}=\xi \beta_{B} J_{0}\left(\beta_{B} r\right) \text {. }
\end{aligned}
$$


Case (ii)

$$
\begin{aligned}
0<\frac{\omega}{c_{1 B}}<\xi< & \frac{\omega}{c_{2 B}}<\infty \\
b_{11} & =\alpha_{B} I_{1}\left(\alpha_{B} r\right) \\
b_{13} & =-\xi J_{1}\left(\beta_{B} r\right) \\
b_{21} & =-\xi I_{0}\left(\alpha_{B} r\right) \\
b_{23} & =\beta_{B} J_{0}\left(\beta_{B} r\right) \\
b_{31} & =\left[\left(\frac{\lambda_{B}}{2 \mu_{B}}+1\right) \alpha_{B}^{2}-\frac{\lambda_{B}}{2 \mu_{B}} \xi^{2}\right] I_{0}\left(\alpha_{B} r\right)-\alpha_{B}^{2} \frac{I_{1}\left(\alpha_{B} r\right)}{\alpha_{B} r} \\
b_{33} & =\xi \beta_{B}\left[-J_{0}\left(\beta_{B} r\right)+\frac{J_{1}\left(\beta_{B} r\right)}{\beta_{B} r}\right] \\
b_{41} & =-\xi \alpha_{B} I_{1}\left(\alpha_{B} r\right) \\
b_{43} & =-\frac{1}{2}\left(\beta_{B}^{2}-\xi^{2}\right) J_{1}\left(\beta_{B} r\right) \\
b_{61} & =\left[\frac{\lambda_{B}}{2 \mu_{B}} \alpha_{B}^{2}-\left(\frac{\lambda_{B}}{2 \mu_{B}}+1\right) \xi^{2}\right] I_{0}\left(\alpha_{B} r\right) \\
b_{63} & =\xi \beta_{B} J_{0}\left(\beta_{B} r\right) . \\
b_{53} & =-\xi \beta_{B} \frac{J_{1}\left(\beta_{B} r\right)}{\beta_{B} r} \\
b_{51} & =\frac{\lambda_{B}}{2 \mu_{B}}\left(\alpha_{B}^{2}-\xi^{2}\right) I_{0}\left(\alpha_{B} r\right)+\alpha_{B}^{2} \frac{I_{1}\left(\alpha_{B} r\right)}{\alpha_{B} r} \\
& \\
b_{B} &
\end{aligned}
$$

Case (iii)

$$
\begin{aligned}
0<\frac{\omega}{c_{1 B}}<\frac{\omega}{c_{2 B}}< & \xi \infty \\
b_{11} & =\alpha_{B} I_{1}\left(\alpha_{B} r\right) \\
b_{13} & =\xi I_{1}\left(\beta_{B} r\right) \\
b_{21} & =-\xi I_{0}\left(\alpha_{B} r\right) \\
b_{23} & =-\beta_{B} I_{0}\left(\beta_{B} r\right)
\end{aligned}
$$




$$
\begin{aligned}
& b_{31}=\left[\left(\frac{\lambda_{B}}{2 \mu_{B}}+1\right) \alpha_{B}^{2}-\frac{\lambda_{B}}{2 \mu_{B}} \xi^{2}\right] I_{0}\left(\alpha_{B} r\right)-\alpha_{B}^{2} \frac{I_{1}\left(\alpha_{B} r\right)}{\alpha_{B} r} \\
& b_{33}=\xi \beta_{B}\left[I_{0}\left(\beta_{B} r\right)-\frac{I_{1}\left(\beta_{B} r\right)}{\beta_{B} r}\right] \\
& b_{41}=-\xi \alpha_{B} I_{1}\left(\alpha_{B} r\right) \\
& b_{43}=-\frac{1}{2}\left(\beta_{B}^{2}+\xi^{2}\right) I_{1}\left(\beta_{B} r\right) \\
& b_{51}=\frac{\lambda_{B}}{2 \mu_{B}}\left(\alpha_{B}^{2}-\xi^{2}\right) I_{0}\left(\alpha_{B} r\right)+\alpha_{B}^{2} \frac{I_{1}\left(\alpha_{B} r\right)}{\alpha_{B} r} \\
& b_{53}=\xi \beta_{B} \frac{I_{1}\left(\beta_{B} r\right)}{\beta_{B} r} \\
& b_{61}=\left[\frac{\lambda_{B}}{2 \mu_{B}} \alpha_{B}^{2}-\left(\frac{\lambda_{B}}{2 \mu_{B}}+1\right) \xi^{2}\right] I_{0}\left(\alpha_{B} r\right) \\
& b_{63}=-\xi \beta_{B} I_{0}\left(\beta_{B} r\right) .
\end{aligned}
$$

\section{References}

Farris T N, Murthy H, Matlik J F 2003 Fretting fatigue, In R O Rithcie and Y Murakami, (eds.), Comprehensive structural integrity Elsevier, Pergamon Press 4: 281-326

Graff K F 1975 Wave motion in elastic loads. (New York: Dover Publications)

Matlik J F, Farris T N 2003 High frequency fretting fatigue experiments. Fretting fatigue: Advances in basic understanding and applications, ASTM STP 1425

Nishida T, Mizutani J, Mutoh Y, Maejima M 2003 Effect of lubricating anodic film on fretting fatigue strength of aluminium alloy. Fretting fatigue: Advances in basic understanding and applications, ASTM STP 1425

Okane M, Shiozawa K, Hiki M, Suzuki K 2003 Fretting fatigue properties of WC-Co Thermal sprayed NiCrMo steel. Fretting fatigue: Advances in basic understanding and applications, ASTM STP 1425

Ramesh M, Satish V Kailas, Simha K R Y 2006 High frequency fretting fatigue in cylinders. Int. Conf. on Industrial Tribol. Nov 30-Dec 02 (Bangalore: Indian Institute of Science)

Rehbein P, Wallaschek J, Lohe D 1999 A test method to investigate the tribological behaviour of friction materials-Ultrasonic fretting conditions. Tribotest $J$.

Soderberg S, Nikoonezhad S, Salama K, Vingsbo O 1986 Accelerated fretting wear testing using ultrasonics. Ultrasonics 24: 348-353

Whittier J S, Jones J P 1967 Axially symmetric wave propagation in two-layered cylinder. Int. J. Solids and Struct. 3: 657-675 